\title{
e-interview
}

\section{David M. Ndetei}

David M. Ndetei is Professor of Psychiatry at the University of Nairobi, Kenya and founder and Director of the Africa Mental Health Foundation, a mental health research organisation. He also founded the Nairobi Psychotherapy Institute, which promotes psychotherapy in Kenya. He was trained in Kenya and in London at the Institute of Psychiatry, Guy's Hospital and the Institute of Psychotherapy. His special interest is in operational research on the common clinical issues in Kenya and development of mental health policies for Kenya. e-mail: mndetei@ mentalhealthafrica.com

What has been the greatest impact of your profession on you personally?

The joy and satisfaction of research and the boundless horizons of new knowledge that it provides.

\section{What are your interests outside of work? \\ Sightseeing and tending to my garden and livestock.}

Who was your most influential trainer, and why?

In Kenya it was Dr. G. Mustafa and Dr.W. Muya for mentoring me, and the late Professor J. Muhangi, an incisive clinician and teacher. In the UK it was Professor Julian Leff, my first contact with UK psychiatry, and Professor J. Watson and Dr. M. Lipsedge, all very effective mentors. At theWorld Health Organization it was Professor Norman Sartorius for exposing me to international psychiatry and research

Which book/text has influenced you most?

The DSM-III - because of its holistic approach to clinical practice.

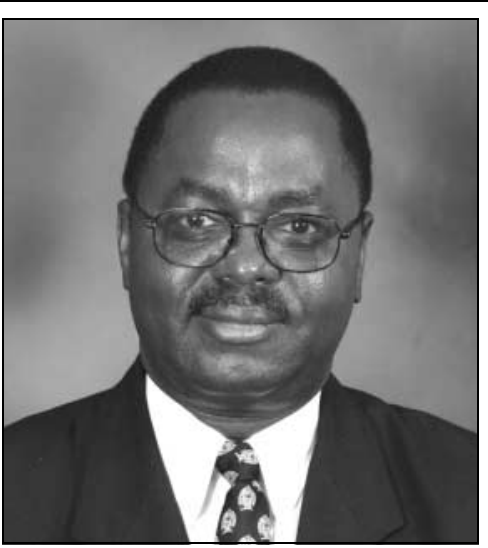

What research publication has had the greatest influence on your work?

Those by the late Professor Lambo on the influence of culture on the manifestation of psychiatric symptoms.

What parts of your work give you the most satisfaction?

Research, teaching, time supervising my students.

What do you least enjoy?

Administration.

What is the most promising opportunity facing the profession? The potential for research to give the profession a stronger scientific basis.

What single change would substantially improve quality of care in Kenya?

A return to family values with the care of the mentally ill in the community, and the empowerment of families and communities through appropriate psycho-education.

\section{What conflict of interest do you} encounter most of ten?

Trying to achieve a balance between research and time spent with my students and the demands of routine administration.
How would you entice more medical students into the profession? Demonstrating leadership by doing rather than saying and at the same time pointing out how to excel.

What is the most important advice you could offer to a new trainee? Do not take anything said as gospel truth - find out for yourself.

What are the main ethical problems that psychiatrists will face in the future?

Using the profession for commercial and political gain or self-promotion, i.e. selfishness.

How would you improve clinical psychiatric training?

By emphasising the bio-psycho-social model approach.

What single change to mental health legislation would you like to see in Kenya?

The prohibition of discrimination on mental health grounds by medical insurance schemes in Kenya.

What is the future for psychotherapy in psychiatry training and practice? Good, as part of the integrated model.

What single area of psychiatric research should be given priority? Operational research to provide costeffective clinical practice and the right mental health policies.

What single area of psychiatric practice is most in need of development?

Liaison psychiatry all the way from tertiary to primary and community healthcare levels.

Dominic Fannon 